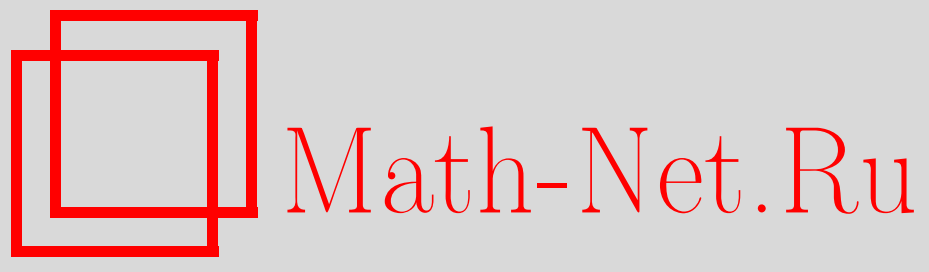

И. С. Резвякова, О нулях дзета-функции Эпштейна на критической прямой, УМН, 2015, том 70, выпуск 4, 213214

DOI: https://doi.org/10.4213/rm9671

Использование Общероссийского математического портала Math-Net.Ru подразумевает, что вы прочитали и согласны с пользовательским соглашением http://www . mathnet.ru/rus/agreement

Параметры загрузки:

IP : 52.90 .164 .192

26 апреля 2023 г., 18:29:03

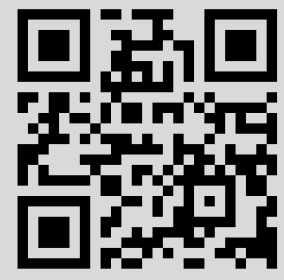




\section{О нулях дзета-функции Эпштейна на критической прямой}

\section{И. С. Резвякова}

Пусть $Q(n, m)=a n^{2}+b n m+c m^{2}-$ положительно определенная квадратичная форма с целыми коэффициентами, причем $-D=b^{2}-4 a c<0$ - фундаментальный дискриминант. Рассмотрим дзета-функцию Эпштейна $\zeta_{Q}(s)$, задающуюся при $\operatorname{Re} s>1$ рядом

$$
\zeta_{Q}(s)=\sum_{(n, m) \neq(0,0)}(Q(n, m))^{-s} .
$$

Известно, что $\zeta_{Q}(s)$ представляется в виде линейной комбинации L-функций с характерами Гекке на группе классов идеалов мнимого квадратичного расширения поля рациональных чисел $\mathbb{Q}(\sqrt{-D})$, а именно,

$$
\zeta_{Q}(s)=\frac{\varepsilon_{-D}}{h(-D)} \sum_{j=1}^{h} \overline{\psi_{j}\left(I_{Q}\right)} L\left(s, \psi_{j}\right),
$$

где $h=h(-D)$ - число классов, $\varepsilon_{-D}$ - число единиц, $I_{Q}=(a, b-i \sqrt{D} / 2)$, a L-функции Гекке задаются следующим эйлеровым произведением по всем простым идеалам:

$$
L\left(s, \psi_{j}\right)=\prod_{\mathfrak{p}}\left(1-\frac{\psi_{j}(\mathfrak{p})}{N \mathfrak{p}^{s}}\right)^{-1},
$$

где $N \mathfrak{p}$ - норма идеала $\mathfrak{p}$. Дзета-функция Эпштейна мероморфно продолжается на всю комплексную плоскость и удовлетворяет функциональному уравнению риманова типа:

$$
\left(\frac{2 \pi}{\sqrt{D}}\right)^{-s} \Gamma(s) \zeta_{Q}(s)=\left(\frac{2 \pi}{\sqrt{D}}\right)^{1-s} \Gamma(1-s) \zeta_{Q}(1-s) .
$$

Таким образом, все комплексные нули $\zeta_{Q}(s)$ симметричны относительно критической прямой $\operatorname{Re} s=1 / 2$, а также относительно вещественной оси. Если $h(-D)>1$, то $\zeta_{Q}(s)$ не имеет эйлерова произведения. В этом случае аналог гипотезы Римана для нее не справедлив и можно доказать, что в любом прямоугольнике вида $\{0<\operatorname{Im} s<T, a \leqslant \operatorname{Re} s \leqslant b\}$, где $1 / 2<a<1$, лежит порядка $T$ нулей (см. [1], [2]). Существует гипотеза, что почти все нетривиальные (т. е. не являющиеся вещественными) нули дзета-функции Эпштейна лежат на критической прямой. Эта гипотеза была условно доказана Э. Бомбьери и Д. Хейчалом в 1987 г. в предположении справедливости гипотезы Римана для L-функций Гекке и ослабленной гипотезы Монтгомери о парной корреляции ординат их комплексных нулей. Нами доказан следующий безусловный результат.

Теорема 1. Положительная доля нетривиалъных нулей $\zeta_{Q}(s)$ лежит на критической прямой $\operatorname{Re} s=1 / 2$.

Эта теорема является одним из продолжений работы А. Сельберга [3], в которой он доказал, что положительная доля нетривиальных нулей дзета-функции Римана лежит на критической прямой. Аналогичные теоремы для более широкого класca L-функций, имеющих эйлерово произведение, можно найти в [4], [5]. Известные ранее результаты (которые по порядку, однако, слабее, чем результат настоящей теоремы) о нулях, лежащих на критической прямой, для некоторых линейных комбинаций L-функций можно найти в работах C. M. Воронина, A. A. Карацубы, С.А. Гриценко и автора. Впервые теорема о положительной доле нулей в случае

Исследование выполнено за счет гранта Российского научного фонда (проект № 14-50-00005).

DOI: $10.4213 / \mathrm{rm} 9671$ 
линейной комбинации L-функций Дирихле появилась в конце 1990-х годов в записях А. Сельберга [6], где были также изложены идеи доказательства. Однако случай дзета-функции Эпштейна, как отмечено в [7], оставался открытым. На основе результатов работы [5] в [8] нами было получено обобщение работы [6] для случая линейной комбинации L-функций Гекке с комплексными характерами. Кроме методов работы [3] А. Сельберг опирался в [6] на предельную теорему для разности логарифмов L-функций на критической прямой. Пример доказательства предельной теоремы для дзета-функции Римана на критической прямой при помощи вероятностного неравенства Берри-Эссеена и оценок моментов из [9] можно найти в [10]. Для доказательства теоремы 1 в случае дзета-функции Эпштейна помимо результатов работы [8] необходимы дополнительные утверждения для L-функций с вещественными характерами Гекке (т. е. L-функций, являющихся произведением двух L-функций Дирихле). Эти утверждения связаны с получением правильной по порядку оценки среднего значения произведения L-функции и соответствующего "успокаивающего" множителя и сводятся в итоге к получению асимптотической формулы в аддитивной проблеме с коэффициентами соответствующей L-функции. Сформулируем некоторые основные утверждения, необходимые для доказательства теоремы 1.

Лемма 1. Пусть $\varkappa_{a, b}$ - характеристическая функиия произвольного интервала $(a, b)$. Для двух различных L-функций Гекке имеет место равенство

$$
\int_{T}^{2 T} \varkappa_{a, b}\left(\frac{\log \left|L_{j}(1 / 2+i t)\right|-\log \left|L_{j^{\prime}}(1 / 2+i t)\right|}{\sqrt{\left(n_{j}+n_{j^{\prime}}\right) \pi \log \log T}}\right) d t=T \int_{a}^{b} e^{-\pi u^{2}} d u+o(T)
$$

при $T \rightarrow+\infty$, где $n_{j}=1$ или 2 в зависимости от того, является характер Гекке комплексным или вещественным.

Лемма 2. Пусть $r(n)$ - коэфбициенты L-функции с вещественным характером Гекке на группе классов идеалов поля $\mathbb{Q}(\sqrt{-D})$, m.е. $r(n)=\sum_{d \mid n} \chi_{v}(d) \chi_{w}(n / d)$ $(v w=D)$ есть свертка двух характеров Дирихле, $\left(m_{1}, m_{2}\right)=1, l$ - натуральное число. Тогда справедливо тождество

$$
\sum_{\substack{m_{2} n_{2}-m_{1} n_{1}=l \\ n_{1}<N}} r\left(n_{1}\right) r\left(n_{2}\right)=c\left(m_{1}, m_{2}, l, v, w\right) N+R,
$$

где остаток $R$ обладает свойством $R \ll_{m_{1}, m_{2}, l} N^{7 / 8+\varepsilon}$ и степенным образом завиcит от параметров $m_{1}, m_{2}, l$, а постоянная $c=c\left(m_{1}, m_{2}, l, v, w\right)$ явно вычисляется.

\section{Список литературы}

[1] H. Davenport, H. Heilbronn, J. Lond. Math. Soc., S1-11:3 (1936), 181-185; S1-11:4 (1936), 307-312. [2] С. М. Воронин, Теория чисел, математический анализ и их приложения, Тр. МИАН СССР, 142, 1976, 135-147. [3] А. Selberg, Skr. Norske Vid. Akad. Oslo I, 1942:10 (1942), 59 pp.. [4] J. L. Hafner, Math. Ann., 264 (1983), 21-37. [5] И. С. Резвякова, Матем. заметки, 88:3 (2010), 456-475. [6] A. Selberg, Linear combinations of L-functions and zeros on the critical line, 1999, 18 pp., http://www.msri.org/realvideo/ln/msri/1999/random/selberg/1/main.html. [7] Э. Бомбьери, А. Гош, УМН, 66:2(398) (2011), 15-66. [8] I. S. Rezvyakova, On the zeros of linear combinations of degree two L-functions on the critical line. Selberg's approach, 2015, 22 pp., DOI: 10.13140/RG.2.1.1488.5281. [9] A. Selberg, Arch. Math. Naturvid., 48:5 (1946), 89-155. [10] A. Ghosh, J. Number Theory, 17:1 (1983), 93-102.

\section{Ирина Сергеевна Резвякова}

(Irina S. Rezvyakova)

Математический институт им. В. А. Стеклова

Российской академии наук

E-mail: irezvyakova@gmail.com, rezvyakova@mi.ras.ru
Представлено Д. О. Орловым Принято редколлегией 05.05 .2015 